\title{
IRREDUCIBLE REPRESENTATIONS OF THE QUANTUM WEYL ALGEBRA AT ROOTS OF UNITY GIVEN BY MATRICES
}

\author{
BLAISE HEIDER AND LINHONG WANG
}

\begin{abstract}
To describe the representation theory of the quantum Weyl algebra at an $l$ th primitive root $\gamma$ of unity, Boyette, Leyk, Plunkett, Sipe, and Talley found all nonsingular irreducible matrix solutions to the equation $y x-\gamma x y=1$, assuming $y x \neq x y$. In this note, we complete their result by finding and classifying, up to equivalence, all irreducible matrix solutions $(X, Y)$, where $X$ is singular.
\end{abstract}

\section{INTRODUCTION}

Let $\mathbb{K}$ be an algebraically closed field, $\gamma$ a nonzero scalar in $\mathbb{K}$. The irreducible representations of the quantized Weyl algebra $\mathbb{A}=\mathbb{K}\{x, y\} /\langle y x-\gamma x y-1\rangle$ has been constructed and classified in [2] and [3]. It is well known that, when $\gamma$ is a root of unity, any irreducible representation of $\mathbb{A}$ is finite dimensional. In [1], Boyette, Leyk, Plunkett, Sipe, and Talley present a linear algebra method to prove the DrozdGuzner-Ovsienko result in the case when $\gamma$ is a primitive root of unity. Let $V$ be an $\mathbb{A}$-module and $\rho: \mathbb{A} \rightarrow \operatorname{End}(V) \hookrightarrow M_{n}(\mathbb{K})$ be a representation of $\mathbb{A}$. Then $\rho$ is irreducible, i.e., $V$ is simple, if and only if $\rho(\mathbb{A})=\mathrm{M}_{n}(\mathbb{K})$. Their approach then is to find explicitly, up to equivalence, all irreducible matrix solutions to the equation $y x-\gamma x y=1$ with $y x \neq x y$. Two $n \times n$ matrices $X$ and $Y$ form a matrix solution if $Y X-\gamma X Y=I$, the identity matrix. A solution $(X, Y)$ is irreducible if every matrix in $M_{n}(\mathbb{K})$ can be written as a noncommutative polynomial in $X$ and $Y$ over $\mathbb{K}$, assuming the zero power of a matrix is the identity matrix. Two solutions $(X, Y)$ and $(M, N)$ are equivalent if there is a nonsingular matrix $Q$ such that $Q X Q^{-1}=M$ and $Q Y Q^{-1}=N$.

Throughout, $\gamma$ is an $l$ th primitive root of unity for some integer $l \geq 2$. Unless specified otherwise, any solution is a matrix solution to the equation $y x-\gamma x y=1$.

1.1. Nonsingular solutions. Suppose $(X, Y)$ is an irreducible solution and $U=$ $Y X-X Y \neq 0$. The following are proved in [1].

(i) $Y X^{l}=X^{l} Y$ and $Y^{l} X=X Y^{l}$, and so $X^{l}$ and $Y^{l}$ are scalar matrices. It follows that any irreducible matrix solutions is at most $l \times l$.

2010 Mathematics Subject Classification. 16D60, 81R50.

Key words and phrases. quantum Weyl algebra, representations, matrix equations.

The first author was supported by an undergraduate research grant from CST at Southeastern Louisiana University. Research of the second author supported by the Louisiana Board of Regents [LEQSF(2012-15)-RD-A-20]. 
(ii) $U X=\gamma X U, Y U=\gamma U Y$, and $U$ is nonsingular. If $X$ has one nonzero eigenvalue, say $\lambda$, then $X$ has at least $l$ distinct eigenvalues, $\gamma \lambda, \gamma^{2} \lambda, \ldots, \gamma^{l} \lambda$. In particular, $(X, Y)$ is at least $l \times l$.

(iii) When $X$ has at least one nonzero eigenvalue, all irreducible solutions are $l \times l$ and found explicitly in [1] as follows.

$$
X_{\lambda}=\lambda\left(\begin{array}{cccc}
\gamma & 0 & \ldots & 0 \\
0 & \gamma^{2} & \ldots & 0 \\
\vdots & \vdots & \ddots & \vdots \\
0 & 0 & \ldots & \gamma^{l}
\end{array}\right) Y_{\lambda b^{\prime} s}=\left(\begin{array}{cccccc}
\frac{1}{(1-\gamma) \gamma \lambda} & b_{1} & 0 & \ldots & 0 & 0 \\
0 & \frac{1}{(1-\gamma) \gamma^{2} \lambda} & b_{2} & \ldots & 0 & 0 \\
& \vdots & \vdots & \ddots & \vdots & \vdots \\
\vdots & 0 & 0 & \ldots & \frac{1}{(1-\gamma) \gamma^{l-1} \lambda} & b_{l-1} \\
0 & 0 & 0 & \ldots & 0 & \frac{1}{(1-\gamma) \gamma^{l} \lambda}
\end{array}\right)
$$

where $\lambda, b$ 's are nonzero scalars in $\mathbb{K}$. The two matrices in each of these solutions are both nonsingular. These solutions are corresponding to the $x$-, $y$-torsion-free simple A-modules. (cf. [3, Summary 3.7])

However, for an irreducible solution $(X, Y)$, it is not always true that $X$ has nonzero eigenvalues. For example, when $l=2, \gamma=-1$, the matrices

$$
X=\left(\begin{array}{cc}
0 & 1 \\
0 & 0
\end{array}\right) \quad \text { and } \quad Y=\left(\begin{array}{cc}
0 & 0 \\
1 & 0
\end{array}\right)
$$

form an irreducible solution to the equation $y x+x y=1$. In fact, this solution gives the only simple $A$-module, where $\mathbb{A}=\mathbb{K}\{x, y\} /\langle y x+x y=1\rangle$, that is both $x$ - and $y$-torsion. (cf. [3, Example 4.1]) Hence, the remark (ii) in [1] and the assertion in [1, Proposition 2] are incorrect.

In this note, we complete [1, Proposition 2] by finding, up to equivalence, all irreducible matrix solutions $(X, Y)$, where $X$ only has zero eigenvalue. Moreover, the two types of irreducible matrix solutions, with $X$ nonsingular or singular, are classified up to equivalence. These irreducible solutions give explicitly all irreducible representations of the quantum Weyl algebra at the root $\gamma$.

Acknowledgement. This problem arose in conversations between the second author and her Ph. D. advisor, E. Letzter. The authors are thankful for his generosity. The authors are also grateful to the referee for the comments that lead to Lemma 2.4.

\section{IRREDUCIBLE MATRIX SOLUTIONS WITH $X$ SINGULAR}

Direct computation shows that the following is a solution. 


\subsection{Solution.}

$$
X=\left(\begin{array}{ccccc}
0 & 1 & & & \\
& 0 & 1 & & \\
& & & \ddots & \\
& & & 0 & 1 \\
& & & & 0
\end{array}\right)_{l \times l} Y=Y_{\beta}=\left(\begin{array}{cccccc}
0 & 0 & \ldots & 0 & 0 & \beta \\
\sum_{i=0}^{l-2} \gamma^{i} & 0 & \ldots & 0 & 0 & 0 \\
0 & \sum_{i=0}^{l-3} \gamma^{i} & \ldots & 0 & 0 & 0 \\
\vdots & \vdots & \ddots & \vdots & \vdots & \vdots \\
0 & 0 & \ldots & 1+\gamma & 0 & 0 \\
0 & 0 & \ldots & 0 & 1 & 0
\end{array}\right)
$$

where $\beta$ is a scalar in $\mathbb{K}$. The following on the matrices $X$ and $Y$ are either standard or straightforward.

(i) Note that $X$ is the well-known upper shift matrix, and $X^{l}=0$. Fix a positive integer $u \leq l-1$. Then $X^{u}$ is a matrix with $u$ th superdiagonal line all ones and zeroes elsewhere. Premultiplying a matrix $A$ by $X^{u}$ results in a matrix whose last $u$ rows are all zeroes and the first $l-u$ rows are the last $l-u$ rows of the matrix $A$.

(ii) Note that each $Y_{k, k-1}$ along the subdiagonal line of $Y$ is the only nonzero entry on the $k$ th row of $Y$. Fix a positive integer $v \leq l-1$. It follows that

$$
\left(Y^{v}\right)_{l, k}= \begin{cases}0 & k \neq l-v \\ \prod_{j=l-v+1}^{l} \sum_{i=0}^{l-j} \gamma^{i} & k=l-v\end{cases}
$$

That is, the only nonzero entry on the $l$ th row of the matrix $Y^{v}$ is $\left(Y^{v}\right)_{l, l-v}$.

2.2. Proposition. The solution $(X, Y)$ in (2.1) is irreducible.

Proof. Let $H=\operatorname{Span}_{\mathbb{K}}\left\{X^{i} Y^{j} ; \quad 0 \leq i, j \leq l-1\right\}$. For any positive integers $m, n \leq l$, we will show that the elementary matrices $\mathbf{e}_{m n}$ are in $H$.

For fixed integers $m, n \leq l$, consider the matrix $X^{l-m} Y^{l-n}$. By $(2.1 \mathrm{i})$, the last nonzero row of $X^{l-m} Y^{l-n}$ is the $m$ th row, which is the same as the $l$ th row of the matrix $Y^{l-n}$. By (2.1 ii), the only nonzero entry in the $l$ th row of $Y^{l-n}$ is

$$
\left(Y^{l-n}\right)_{l, n}=\prod_{j=n+1}^{l} \sum_{i=0}^{l-j} \gamma^{i}
$$

Hence,

$$
X^{l-m} Y^{l-n}=\left(\prod_{j=n+1}^{l} \sum_{i=0}^{l-j} \gamma^{i}\right) \mathbf{e}_{m n}+\left(\sum_{s=1}^{m-1} \sum_{t=1}^{l}\left(X^{l-m} Y^{l-n}\right)_{s, t}\right) \mathbf{e}_{s t}
$$

When $m=1$, we have

$$
X^{l-1} Y^{l-n}=\left(\prod_{j=n+1}^{l} \sum_{i=0}^{l-j} \gamma^{i}\right) \mathbf{e}_{1 n}
$$

for $n=1, \ldots, l$. Then, by induction on $m$, it shows that any $\mathbf{e}_{m n} \in H$. 
2.3. Proposition. Suppose $(A, B)$ with $B A \neq A B$ is an irreducible solution. If the only eigenvalue of $A$ is 0 , then $(A, B)$ is equivalent to a solution as follows.

$$
X=\left(\begin{array}{ccccc}
0 & 1 & & & \\
& 0 & 1 & & \\
& & & \ddots & \\
& & & 0 & 1 \\
& & & & 0
\end{array}\right)_{l \times l} Y_{\alpha^{\prime} s}=\left(\begin{array}{cccccc}
\gamma^{l-1} \alpha_{l} & \gamma^{l-2} \alpha_{l-1} & \ldots & \gamma^{2} \alpha_{3} & \gamma \alpha_{2} & \alpha_{1} \\
\sum_{i=0}^{l-2} \gamma^{i} & \gamma^{l-2} \alpha_{l} & \ldots & \gamma^{2} \alpha_{4} & \gamma \alpha_{3} & \alpha_{2} \\
0 & \sum_{i=0}^{l-3} \gamma^{i} & \ldots & \gamma^{2} \alpha_{5} & \gamma \alpha_{4} & \alpha_{3} \\
\vdots & \vdots & \ddots & \vdots & \vdots & \vdots \\
0 & 0 & \ldots & 1+\gamma & \gamma \alpha_{l} & \alpha_{l-1} \\
0 & 0 & \ldots & 0 & 1 & \alpha_{l}
\end{array}\right)
$$

where $\alpha_{i}$ are scalars in $\mathbb{K}$ for $i=1,2, \ldots, l$.

Proof. Suppose that $(A, B)$ with $B A \neq A B$ is an $n \times n$ irreducible solution for some positive integer $n$ and that the only eigenvalue of $A$ is 0 . Let $C=Q A Q^{-1}$ be the Jordan Canonical form for $A$, where $Q$ is an $n \times n$ matrix. Set $D=Q B Q^{-1}$. Then $(C, D)$ is also an irreducible solution.

Suppose the Jordan blocks $J_{i}$ in $C$ are $m_{i} \times m_{i}$ for $i=1, \ldots, k$. Partition the matrix $D$ into blocks $\left(D_{i j}\right)$, where $D_{i j}$ are $m_{i} \times m_{j}$ matrices, for $1 \leq i, j \leq k$. Then we have $D_{i i} J_{i}-\gamma J_{i} D_{i i}=1$ for $i=1,2, \ldots, k$. Fix $i$, let $D_{i i}=\left(d_{p q}\right)_{m_{i} \times m_{i}}$. Note that along the diagonal line of the matrix $D_{i i} J_{i}-\gamma J_{i} D_{i i}$ should be all ones, i.e.,

$$
1=0-\gamma d_{21}, 1=d_{21}-\gamma d_{32}, \ldots, 1=d_{m_{i}-1 m_{i}-2}-\gamma d_{m_{i} m_{i}-1} \text {, and } 1=d_{m_{i} m_{i}-1} .
$$

It then follows that $1+\gamma+\gamma^{2}+\ldots+\gamma^{m_{i}-1}=0$. But $\gamma$ is an $l$ th primitive root of unity. Thus $m_{i}$ must be an integer multiple of $l$. Hence, $n=\sum_{i} m_{i}$ must be greater than or equal to $l$. It then follows from (1.1, i) that $C$ has to be the $l \times l$ Jordan block with zeroes on the diagonal line, i.e., $C=Q A Q^{-1}=X$.

Now, it is sufficient to show that $D=Q B Q^{-1}$ must have the form $Y_{\alpha^{\prime} \mathrm{s}}$. It follows from $B A-\gamma A B=1$ that $D X-\gamma X D=1$. We will explore the problem element-wise. By $(2.1$, i), we have

$$
(D X)_{i j}=\left\{\begin{array}{ll}
d_{i j-1}, & j>1 \\
0, & j=1
\end{array} \quad \text { and } \quad(\gamma X D)_{i j}= \begin{cases}\gamma d_{i+1 j} & i<l \\
0, & i=l\end{cases}\right.
$$

Since $i+1 \neq 1$ and $j-1 \neq l$, it is clear that $d_{1 l}$ is a free variable.

Case $1, i<j$. Fix $1 \leq k \leq l-1$, the $k$ th superdiagonal line of $D X-\gamma X D$ has entries

$$
(D X-\gamma X D)_{i, i+k}=d_{i, i+k-1}-\gamma d_{i+1, i+k} \quad \text { for } \quad i=1,2, \ldots, l-k .
$$

That is, $d_{1, k-1}-\gamma d_{2 k}=0, d_{2 k}-\gamma d_{3, k+1}=0 \ldots d_{l-k, l-1}-\gamma d_{l-k+1, l}=0$. Then, inductively,

$$
d_{i, i+k-1}=\gamma^{l-k} d_{l-k+1, l} \quad \text { for } \quad i=1,2, \ldots, l-k,
$$

Therefore, on the $l$ th column of $D$, we get free variables $d_{2 l}, \ldots, d_{l-1, l}$. 
Case $2, i=j$. We have

$$
1=(D X-\gamma X D)_{i i}= \begin{cases}0-\gamma d_{21} & i=1 \\ d_{i, i-1}-\gamma d_{i+1, i} & 1<i<l \\ d_{l, l-1}-0 & i=l\end{cases}
$$

Thus, the entries on the subdiagonal line of $D$ are

$$
d_{21}=-\frac{1}{\gamma}=\sum_{i=0}^{l-2} \gamma^{i}, \quad d_{32}=\frac{d_{21}-1}{\gamma}=\sum_{i=0}^{l-3} \gamma^{i}, \quad \ldots, \quad d_{l-1, l-2}=1+\gamma, \text { and } d_{l, l-1}=1
$$

Case $3, i>j$. We have

$$
0=(D X-\gamma X D)_{i j}= \begin{cases}0-\gamma d_{i+1,1} & j=l \text { and } j<i<l \\ d_{l, j-1} & i=l \text { and } 1<j<i \\ d_{i, j-1}-\gamma d_{i+1, j} & i<l \text { and } 1<j<i\end{cases}
$$

It is not hard to see, inductively, that $d_{i j}=0$ for any $2<i \leq l$ and $1 \leq j \leq i-2$. The proposition follows.

2.4. Lemma. Any solution $\left(X, Y_{\alpha^{\prime} s}\right)$ in (2.3) is equivalent to a solution $\left(X, Y_{\beta}\right)$ in (2.1). The equivalence classes of solutions in (2.3) are $\left[\left(X, Y_{\beta}\right)\right]$ for $\beta \in \mathbb{K}$.

Proof. Suppose $\left(X, Y_{\beta}\right)$ is a solution as in (2.1). Direct computation shows that the following uppertriangular matrix $P$ is a nonsingular matrix such that $P^{-1} X P=X$.

$$
P=\left(\begin{array}{cccccc}
1 & p_{l-1} & p_{l-2} & \ldots & p_{2} & p_{1} \\
& 1 & p_{l-1} & \ldots & p_{3} & p_{2} \\
& & & \ddots & & \\
& & & & 1 & p_{l-1} \\
& & & & & 1
\end{array}\right)_{l \times l}
$$

where $p_{k}$ are scalars in $\mathbb{K}$ for $k=1, \ldots, l-1$. Then the matrices $X$ and $P^{-1} Y_{\beta} P$ also form a solution. It is shown in the proof of (2.3) that if $(X, D)$ is a solution then $D$ must be one of the form $Y_{\alpha^{\prime} \mathrm{s}}$. Thus, $P^{-1} Y_{\beta} P=Y_{\alpha^{\prime} \mathrm{s}}$ for some $\alpha_{1}, \ldots, \alpha_{l}$. Consider the matrices $P Y_{\alpha^{\prime} \mathrm{s}}$ and $Y_{\beta} P$. The second row of $Y_{\beta} P$ is

$$
\sum_{i=0}^{l-2} \gamma^{i} \cdot\left(\begin{array}{llll}
1 & p_{l-1} & \cdots & p_{1}
\end{array}\right)
$$

Set $\bar{Y}_{\alpha^{\prime} \mathrm{s}}$ be the lower right $(l-1) \times(l-1)$ block of the matrix $Y_{\alpha^{\prime} \mathrm{s}}$ and $R$ be the $(l-1) \times l$ matrix obtained by removing the last row of the matrix $X$. Then the second row of $P Y_{\alpha^{\prime} \mathrm{s}}$ can be written as

$$
\left(\begin{array}{lllll}
0 & 1 & p_{l-1} & \ldots & p_{2}
\end{array}\right) \cdot Y_{\alpha^{\prime} \mathrm{s}}=\left(\begin{array}{lllll}
\sum_{i=0}^{l-2} \gamma^{i} & 0 & \ldots & 0
\end{array}\right)+\left(\begin{array}{llll}
1 & p_{l-1} & \ldots & p_{2}
\end{array}\right) \cdot \bar{Y}_{\alpha^{\prime} \mathrm{s}} R
$$


It then follows from $P Y_{\alpha^{\prime} \mathrm{s}}=Y_{\beta} P$ that

$$
\sum_{i=0}^{l-2} \gamma^{i} \cdot\left(\begin{array}{lll}
p_{l-1} & \ldots & p_{1}
\end{array}\right)=\left(\begin{array}{llll}
1 & p_{l-1} & \ldots & p_{2}
\end{array}\right) \cdot \bar{Y}_{\alpha^{\prime} \mathrm{s}}
$$

Note that $\bar{Y}_{\alpha^{\prime} \mathrm{s}}$ is the sum of the upper triangular matrix

$$
M=\left(\begin{array}{ccccc}
\gamma^{l-2} \alpha_{l} & \gamma^{l-3} \alpha_{l-1} & \ldots & \gamma \alpha_{3} & \alpha_{2} \\
0 & \gamma^{l-3} \alpha_{l} & \ldots & \gamma \alpha_{4} & \alpha_{3} \\
\vdots & \vdots & \ddots & \vdots & \vdots \\
0 & 0 & \ldots & \gamma \alpha_{l} & \alpha_{l-1} \\
0 & 0 & \ldots & 0 & \alpha_{l}
\end{array}\right)
$$

and the $(l-1) \times(l-1)$ subdiagonal matrix $L$ with entries $\sum_{i=0}^{l-3} \gamma^{i}, \ldots, 1+\gamma, 1$ along the diagonal line. Then we have

$$
\sum_{i=0}^{l-2} \gamma^{i} \cdot\left(\begin{array}{llll}
1 & p_{l-1} & \ldots & p_{1}
\end{array}\right)-\left(\begin{array}{lllll}
1 & p_{l-1} & \ldots & p_{2}
\end{array}\right) \cdot L=\left(\begin{array}{llll}
1 & p_{l-1} & \ldots & p_{2}
\end{array}\right) \cdot M
$$

and so

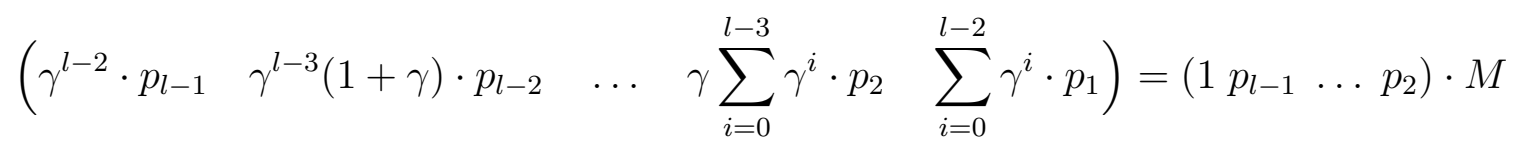

Therefore

$(*)$

$$
\left\{\begin{array}{l}
p_{l-1}=\alpha_{l} \\
p_{k}=\left(\sum_{i=0}^{l-1-k} \gamma^{i}\right)^{-1} \cdot\left(\alpha_{k+1}+\sum_{i=k+1}^{l-1} \alpha_{l+k+1-i} \cdot p_{i}\right) \text { for } k=l-2, \ldots, 1
\end{array}\right.
$$

Moreover, it follows from $\left(P Y_{\alpha^{\prime} \mathrm{s}}\right)_{1, l}=\left(Y_{\beta} P\right)_{1, l}$ that

$$
\beta=\alpha_{1}+\sum_{i=1}^{l-1} \alpha_{l+1-i} \cdot p_{i}
$$

This shows that any solution $\left(X, Y_{\alpha^{\prime} \mathrm{s}}\right)$ is equivalent to a solution $\left(X, Y_{\beta}\right)$ for some $\beta$ in $\mathbb{K}$. The equivalence condition is given by a polynomial condition on $\alpha$ 's and $\beta$ that can be obtained inductively from $(*)$ and $(* *)$. Simply arguing by determinant, we have the equivalence classes are $\left[\left(X, Y_{\beta}\right)\right]$ for $\beta \in \mathbb{K}$.

The preceding lemma shows that, up to equivalence, the only irreducible solution with both determinants equal to zero is the solution $\left(X, Y_{0}\right)$. This solution corresponds to the only $x$ - and $y$-torsion simple module $L(0)$ over the quantum Weyl algebra $\mathbb{A}$ at the root $\gamma$. (cf. [3, Example 4.1]) Next, we provide the equivalence classification for irreducible solutions found in [1], which are corresponding to the $x$ and $y$-torsion-free simple $\mathbb{A}$-modules. 
2.5. Lemma. Any solution $\left(X_{\lambda}, Y_{\lambda b^{\prime} s}\right)$ in (1.1 iii) is equivalent to a solution $\left(X_{\lambda}, Y_{\lambda \eta}\right)$ where

$$
Y_{\lambda \eta}=\left(\begin{array}{cccccc}
\frac{1}{(1-\gamma) \gamma \lambda} & 1 & 0 & \ldots & 0 & 0 \\
0 & \frac{1}{(1-\gamma) \gamma^{2} \lambda} & 1 & \ldots & 0 & 0 \\
\vdots & \vdots & \vdots & \ddots & \vdots & \vdots \\
0 & 0 & 0 & \ldots & \frac{1}{(1-\gamma) \gamma^{l-1} \lambda} & 1 \\
\eta & 0 & 0 & \ldots & 0 & \frac{1}{(1-\gamma) \gamma^{l} \lambda}
\end{array}\right)
$$

for some $\eta \in \mathbb{K}^{\times}$. The equivalence classes of solutions in (1.1 iii) are $\left[\left(X_{\lambda}, Y_{\lambda \eta}\right)\right]$ where $\eta \in \mathbb{K}^{\times}$and $\lambda \in \mathbb{K}^{\times} /\langle\gamma\rangle$.

Proof. Let $\left(X_{\lambda}, Y_{\lambda b^{\prime} s}\right)$ and $\left(X_{\lambda^{\prime}}, Y_{\lambda^{\prime} c^{\prime} s}\right)$ be two irreducible solutions as in (1.1 iii). They are equivalent only if $\lambda^{\prime l}=\lambda^{l}$ and $\prod_{i=1}^{l} b_{i}=\prod_{i=1}^{l} c_{i}$. Suppose $\lambda^{\prime}=\gamma^{i} \lambda$ for some $1 \leq i \leq l$. Then, by using an appropriate permutation matrix, we have $\left(X_{\gamma^{i} \lambda}, Y_{\gamma^{i} \lambda b^{\prime} s}\right)$ is equivalent to $\left(X_{\lambda}, Y_{\lambda b^{\prime} s}\right)$, where the entries $b$ 's in $Y_{\gamma^{i} \lambda b^{\prime} s}$ and those in $Y_{\lambda b^{\prime} s}$ are the same, up to the corresponding permutation. It then remains to show that $\left(X_{\lambda}, Y_{\lambda b^{\prime} s}\right)$ is equivalent to $\left(X_{\lambda}, Y_{\lambda \eta}\right)$, where $\eta=\prod_{i=1}^{l} b_{i}$. This can be done by using a diagonal matrix $P$ with entries

$$
1, b_{1}, b_{1} b_{2}, \ldots, b_{1} \cdots b_{l-1}
$$

along the diagonal line.

Combining Proposition 2.3, Lemma 2.4, (1.1 iii) and Lemma 2.5, we have

2.6. Proposition. ([2, Theorem 5.8]) Any irreducible solution $(A, B)$ to the equation $y x-\gamma x y=1$, in which $B A \neq A B$, is equivalent to either the solution $\left(X_{\lambda}, Y_{\lambda \eta}\right)$ in (2.5) or the solution $\left(X, Y_{\beta}\right)$ in (2.1).

\section{REFERENCES}

[1] J. Boyette, M. Leyk, T. Plunkett, K. Sipe, J. Talley, Explicit representation theory of the quantum Weyl algebra at roots of 1. Comm. Algebra 28 (2000), no. 11, 5269-5274.

[2] Y. A. Drozd, B. L. Guzner, and S. A. Ovsienko, Weight modules over generalized Weyl algebras, J. Algebra 184 (1996), 491-504.

[3] D. A. Jordan, Finite-dimensional simple modules over certain iterated skew polynomial rings, J. Pure Appl. Algebra 98 (1995), 4555.

Department of Mathematics, Southeastern Louisiana University, Hammond, LA 70402

E-mail address: Blaise.Heider@selu.edu, lwang@selu.edu 